\title{
Homoclinic orbits for discrete Hamiltonian systems with subquadratic potential
}

\author{
Xiaoyan Lin ${ }^{*}$ and Xianhua Tang ${ }^{2}$
}

"Correspondence:
xiaoyanlin98@hotmail.com
${ }^{1}$ Department of Mathematics,
Huaihua College, Huaihua, Hunan
418008, P.R. China
Full list of author information is
available at the end of the article

available at the end of the article

\begin{abstract}
In the present paper, we deal with the existence and multiplicity of homoclinic solutions of the second-order self-adjoint discrete Hamiltonian system

$$
\triangle[p(n) \Delta u(n-1)]-L(n) u(n)+\nabla W(n, u(n))=0
$$

Under the assumption that $W(n, x)$ is of indefinite sign and subquadratic as $|x| \rightarrow+\infty$ and $p(n)$ and $L(n)$ are $\mathcal{N} \times \mathcal{N}$ real symmetric positive definite matrices for all $n \in \mathbb{Z}$, and that

$$
\liminf _{|n| \rightarrow+\infty}\left[|n|^{\nu-2} \inf _{|x|=1}(L(n) x, x)\right]>0
$$

for some constant $v<2$, we establish some existence criteria to guarantee that the above system has at least one or multiple homoclinic solutions by using Clark's theorem in critical point theory.
\end{abstract}

MSC: $39 \mathrm{~A} 11 ; 58 \mathrm{E} 05 ; 70 \mathrm{HO}$

Keywords: homoclinic solution; discrete Hamiltonian system; critical point; Clark's theorem

\section{Introduction}

Consider the second-order self-adjoint discrete Hamiltonian system

$$
\triangle[p(n) \triangle u(n-1)]-L(n) u(n)+\nabla W(n, u(n))=0,
$$

where $n \in \mathbb{Z}, u \in \mathbb{R}^{\mathcal{N}}, \Delta u(n)=u(n+1)-u(n)$ is the forward difference operator, $p, L$ : $\mathbb{Z} \rightarrow \mathbb{R}^{\mathcal{N} \times \mathcal{N}}$ and $W: \mathbb{Z} \times \mathbb{R}^{\mathcal{N}} \rightarrow \mathbb{R}$. As usual, we say that a solution $u(n)$ of system (1.1) is homoclinic (to 0 ) if $u(n) \rightarrow 0$ as $n \rightarrow \pm \infty$. In addition, if $u(n) \not \equiv 0$, then $u(n)$ is called a nontrivial homoclinic solution.

In general, system (1.1) may be regarded as a discrete analogue of the following second order Hamiltonian system:

$$
\frac{d}{d t}(p(t) \dot{u}(t))-L(t) u(t)+\nabla W(t, u(t))=0
$$

Moreover, system (1.1) does have its applicable setting as evidenced by monographs [1, 2]. System (1.2) can also be regarded as a special form of the Emden-Fowler equation ap- 
pearing in the study of astrophysics, gas dynamics, fluid mechanics, relativistic mechanics, nuclear physics and chemically reacting system, and many well-known results concerning properties of solutions of (1.2) are collected in [3].

In papers [4-8], the authors studied the existence of homoclinic solutions of system (1.1) or some of its special forms under the following superquadratic growth (AR)-condition on $W$ : there is a constant $\mu>2$ such that

$$
0<\mu W(n, x) \leq(\nabla W(n, x), x), \quad \forall(n, x) \in \mathbb{Z} \times \mathbb{R}^{\mathcal{N}} \backslash\{0\},
$$

or other superquadratic growth conditions, where and in the sequel, (., .) denotes the standard inner product in $\mathbb{R}^{\mathcal{N}}$ and $|\cdot|$ is the induced norm.

When $W(n, x)$ is of subquadratic growth at infinity, Tang and Lin [9] recently established the following results on the existence of homoclinic solutions of system (1.1).

Theorem A [9] Assume that $p(n)$ is an $\mathcal{N} \times \mathcal{N}$ real symmetric positive definite matrix for all $n \in \mathbb{Z}$, and that $L$ and $W$ satisfy the following assumptions:

(L) $L(n)$ is an $\mathcal{N} \times \mathcal{N}$ real symmetric positive definite matrix for all $n \in \mathbb{Z}$, and there exists a constant $\beta>0$ such that

$$
(L(n) x, x) \geq \beta|x|^{2}, \quad \forall(n, x) \in \mathbb{Z} \times \mathbb{R}^{\mathcal{N}}
$$

(W1) For every $n \in \mathbb{Z}, W$ is continuously differentiable in $x$, and there exist two constants $1<\gamma_{1}<\gamma_{2}<2$ and two functions $a_{1}, a_{2} \in l^{2 /\left(2-\gamma_{1}\right)}(\mathbb{Z},[0,+\infty))$ such that

$$
|W(n, x)| \leq a_{1}(n)|x|^{\gamma_{1}}, \quad \forall(n, x) \in \mathbb{Z} \times \mathbb{R}^{\mathcal{N}},|x| \leq 1
$$

and

$$
|W(n, x)| \leq a_{2}(n)|x|^{\gamma_{2}}, \quad \forall(n, x) \in \mathbb{Z} \times \mathbb{R}^{\mathcal{N}},|x| \geq 1
$$

(W2) There exist two functions $b \in l^{2 /\left(2-\gamma_{1}\right)}(\mathbb{Z},[0,+\infty))$ and $\varphi \in C([0,+\infty),[0,+\infty))$ such that

$$
|\nabla W(n, x)| \leq b(n) \varphi(|x|), \quad \forall(n, x) \in \mathbb{Z} \times \mathbb{R}^{\mathcal{N}}
$$

where $\varphi(s)=O\left(s^{\gamma_{1}-1}\right)$ as $s \rightarrow 0^{+}$;

(W3) There exist an $n_{0} \in \mathbb{Z}$ and two constants $\eta>0$ and $\gamma_{3} \in(1,2)$ such that

$$
W\left(n_{0}, x\right) \geq \eta|x|^{\gamma_{3}}, \quad \forall x \in \mathbb{R}^{\mathcal{N}},|x| \leq 1
$$

Then system (1.1) possesses at least one nontrivial homoclinic solution.

Theorem B [9] Assume that $p(n)$ is an $\mathcal{N} \times \mathcal{N}$ real symmetric positive definite matrix for all $n \in \mathbb{Z}$, and that $L$ and $W$ satisfy $(\mathrm{L}),(\mathrm{W} 1),(\mathrm{W} 2)$ and the following assumptions:

(W4) There exist two constants $\eta>0$ and $\gamma_{3} \in(1,2)$ and a set $J \subset \mathbb{Z}$ with $m>0$ elements such that

$$
W(n, x) \geq \eta|x|^{\gamma_{3}}, \quad \forall(n, x) \in J \times \mathbb{R}^{\mathcal{N}},|x| \leq 1
$$


(W5) $W(n,-x)=W(n, x), \forall(n, x) \in \mathbb{Z} \times \mathbb{R}^{\mathcal{N}}$.

Then system (1.1) possesses at least $m$ distinct pairs of nontrivial homoclinic solutions.

When $L(n)$ satisfies (L) in Theorems A and B, assumption (W1) is optimal in some sense, essentially, the summable functions $a_{1}, a_{2} \in l^{2 /\left(2-\gamma_{1}\right)}(\mathbb{R},[0,+\infty))$ are necessary; see Lemma 2.2 in Section 2.

Now a natural question is whether the conditions on the potential $W(n, x)$ can be further relaxed when one imposes stronger conditions on $L(n)$ ?

In the present paper, we give a positive answer to the above question. In fact, we employ Clark's theorem in critical point theory to establish new existence criteria to guarantee that system (1.1) has at least one or multiple homoclinic solutions under the following assumption instead of $(\mathrm{L})$ :

$\left(\mathrm{L}_{v}\right) L(n)$ is an $\mathcal{N} \times \mathcal{N}$ real symmetric positive definite matrix for all $n \in \mathbb{Z}$, and there exists a constant $v<2$ such that

$$
\liminf _{|n| \rightarrow+\infty}\left[|n|^{\nu-2} \inf _{|x|=1}(L(n) x, x)\right]>0 .
$$

Our main results are the following two theorems.

Theorem 1.1 Assume that $p(n)$ is an $\mathcal{N} \times \mathcal{N}$ real symmetric positive definite matrix for all $n \in \mathbb{Z}$, that $L$ satisfies $\left(\mathrm{L}_{v}\right)$ and $W$ satisfies the following assumptions:

(W1') There exist constants $\max \{1,2 /(3-v)\}<\gamma_{1}<\gamma_{2}<2$ and $a_{1}, a_{2} \geq 0$ such that

$$
|W(n, x)| \leq a_{1}|x|^{\gamma_{1}}+a_{2}|x|^{\gamma_{2}}, \quad \forall(n, x) \in \mathbb{Z} \times \mathbb{R}^{\mathcal{N}}
$$

(W2') There exists a function $\varphi \in C([0,+\infty),[0,+\infty))$ such that

$$
|\nabla W(n, x)| \leq \varphi(|x|), \quad \forall(n, x) \in \mathbb{Z} \times \mathbb{R}^{\mathcal{N}}
$$

where $\varphi(s)=O\left(s^{\gamma_{3}-1}\right)$ as $s \rightarrow 0^{+}, \max \{1,2 /(3-v)\}<\gamma_{3}<2$;

$\left(\mathrm{W} 3^{\prime}\right)$ There exist $n_{0} \in \mathbb{Z}$ and constants $\delta_{0}, \eta>0$ and $\max \{1,2 /(3-v)\}<\gamma_{4}<2$ such that

$$
W\left(n_{0}, x\right) \geq \eta|x|^{\gamma_{4}}, \quad \forall x \in \mathbb{R}^{\mathcal{N}},|x| \leq \delta_{0} .
$$

Then system (1.1) possesses at least one nontrivial homoclinic solution.

Theorem 1.2 Assume that $p(n)$ is an $\mathcal{N} \times \mathcal{N}$ real symmetric positive definite matrix for all $n \in \mathbb{Z}$, and that $L$ and $W$ satisfy $\left(\mathrm{L}_{v}\right),\left(\mathrm{W} 1^{\prime}\right),\left(\mathrm{W} 2^{\prime}\right),(\mathrm{W} 5)$ and the following assumption:

(W4') There exist constants $\delta_{0}, \eta>0$ and $\max \{1,2 /(3-v)\}<\gamma_{4}<2$ and a set $J \subset \mathbb{Z}$ with $m>0$ elements such that

$$
W(n, x) \geq \eta|x|^{\gamma_{4}}, \quad \forall(n, x) \in J \times \mathbb{R}^{\mathcal{N}},|x| \leq \delta_{0} .
$$

Then system (1.1) possesses at least $m$ distinct pairs of nontrivial homoclinic solutions. 
Remark 1.3 Obviously, assumptions (W1'), (W2'), (W3') and $\left(\mathrm{W} 4^{\prime}\right)$ are weaker than (W1), (W2), (W3) and (W4), respectively.

The remainder of this paper is organized as follows. In Section 2, some preliminary results are presented. In Section 3, we give the proofs of our theorems. In Section 4, we give some examples to illustrate our results.

\section{Preliminaries}

In this section, we always assume that $p(n)$ and $L(n)$ are real symmetric positive definite matrices for all $n \in \mathbb{Z}$. Let

$$
\begin{aligned}
& S=\left\{\{u(n)\}_{n \in \mathbb{Z}}: u(n) \in \mathbb{R}^{\mathcal{N}}, n \in \mathbb{Z}\right\}, \\
& E=\left\{u \in S: \sum_{n \in \mathbb{Z}}[(p(n+1) \Delta u(n), \Delta u(n))+(L(n) u(n), u(n))]<+\infty\right\},
\end{aligned}
$$

and for $u, v \in E$, let

$$
\langle u, v\rangle=\sum_{n \in \mathbb{Z}}[(p(n+1) \Delta u(n), \Delta v(n))+(L(n) u(n), v(n))] .
$$

Then $E$ is a Hilbert space with the above inner product, and the corresponding norm is

$$
\|u\|=\left\{\sum_{n \in \mathbb{Z}}[(p(n+1) \Delta u(n), \Delta u(n))+(L(n) u(n), u(n))]\right\}^{1 / 2}, \quad u \in E .
$$

As usual, for $1 \leq q<+\infty$, set

$$
l^{q}\left(\mathbb{Z}, \mathbb{R}^{\mathcal{N}}\right)=\left\{\{u(n)\}_{n \in \mathbb{Z}}: u(n) \in \mathbb{R}^{\mathcal{N}}, n \in \mathbb{Z}, \sum_{n \in \mathbb{Z}}|u(n)|^{q}<+\infty\right\}
$$

and

$$
l^{\infty}\left(\mathbb{Z}, \mathbb{R}^{\mathcal{N}}\right)=\left\{\{u(n)\}_{n \in \mathbb{Z}}: u(n) \in \mathbb{R}^{\mathcal{N}}, n \in \mathbb{Z}, \sup _{n \in \mathbb{Z}}|u(n)|<+\infty\right\}
$$

and their norms are defined by

$$
\begin{aligned}
& \|u\|_{q}=\left(\sum_{n \in \mathbb{Z}}|u(n)|^{q}\right)^{1 / q}, \quad \forall u \in l^{q}\left(\mathbb{Z}, \mathbb{R}^{\mathcal{N}}\right) ; \\
& \|u\|_{\infty}=\sup _{n \in \mathbb{Z}}|u(n)|, \quad \forall u \in l^{\infty}\left(\mathbb{Z}, \mathbb{R}^{\mathcal{N}}\right),
\end{aligned}
$$

respectively.

Lemma 2.1 [9] For $u \in E$,

$$
\|u\|_{\infty} \leq \frac{1}{\sqrt[4]{(\beta+4 \alpha) \beta}}\|u\|
$$

where $\alpha=\inf \left\{(p(n) x, x): n \in \mathbb{Z}, x \in \mathbb{R}^{\mathcal{N}},|x|=1\right\}$ and $\beta=\inf \left\{(L(n) x, x): n \in \mathbb{Z}, x \in \mathbb{R}^{\mathcal{N}}\right.$, $|x|=1\}$. 
By $\left(\mathrm{L}_{v}\right)$, there exist two constants $N_{0}>0$ and $M_{0}>0$ such that

$$
|n|^{\nu-2} \inf _{|x|=1}(L(n) x, x) \geq M_{0}, \quad|n| \geq N_{0}
$$

which implies

$$
|n|^{\nu-2}(L(n) x, x) \geq M_{0}|x|^{2}, \quad|n| \geq N_{0}, x \in \mathbb{R}^{\mathcal{N}} .
$$

Lemma 2.2 Suppose that L satisfies $\left(\mathrm{L}_{v}\right)$. Then, for $1 \leq q \in(2 /(3-v), 2), E$ is compactly embedded in $l^{q}\left(\mathbb{Z}, \mathbb{R}^{\mathcal{N}}\right)$; moreover,

$$
\sum_{|n|>N}|u(n)|^{q} \leq \frac{K(q)}{N^{\kappa}}\|u\|^{q}, \quad \forall u \in E, N \geq N_{0}
$$

and

$$
\|u\|_{q}^{q} \leq\left[\left(\sum_{|n| \leq N}[l(n)]^{-q /(2-q)}\right)^{1-\frac{q}{2}}+\frac{K(q)}{N^{\kappa}}\right]\|u\|^{q}, \quad \forall u \in E, N \geq N_{0},
$$

where

$$
\kappa=\frac{(3-v) q-2}{2}>0, \quad K(q)=\left[\frac{2(2-q)}{(3-v) q-2}\right]^{1-\frac{q}{2}} M_{0}^{-q / 2}
$$

and

$$
l(n)=\inf _{x \in \mathbb{R}^{\mathcal{N}},|x|=1}(L(n) x, x)
$$

Proof Let $r=[(3-v) q-2] /(2-q)$. Then $r>0$. For $u \in E$ and $N \geq N_{0}$, it follows from (2.2), (2.5) and the Hölder inequality that

$$
\begin{aligned}
\sum_{|n|>N}|u(n)|^{q} & \leq\left(\sum_{|n|>N}|n|^{-(2-v) q /(2-q)}\right)^{1-\frac{q}{2}}\left(\sum_{|n|>N}|n|^{2-v}|u(n)|^{2}\right)^{\frac{q}{2}} \\
& \leq\left(\frac{2}{r N^{r}}\right)^{1-\frac{q}{2}}\left[\frac{1}{M_{0}} \sum_{|n|>N}(L(n) u(n), u(n))\right]^{\frac{q}{2}} \\
& \leq \frac{2^{(2-q) / 2}}{M_{0}^{q / 2} r^{(2-q) / 2} N^{\kappa}}\|u\|^{q}=\frac{K(q)}{N^{\kappa}}\|u\|^{q} .
\end{aligned}
$$

This shows that (2.3) holds. Hence, from (2.2), (2.6) and the Hölder inequality, one has

$$
\begin{aligned}
\|u\|_{q}^{q} & =\sum_{|n| \leq N}|u(n)|^{q}+\sum_{|n|>N}|u(n)|^{q} \\
& \leq\left(\sum_{|n| \leq N}[l(n)]^{-q /(2-q)}\right)^{1-\frac{q}{2}}\left(\sum_{|n| \leq N} l(n)|u(n)|^{2}\right)^{\frac{q}{2}}+\frac{K(q)}{N^{\kappa}}\|u\|^{q} \\
& \leq\left(\sum_{|n| \leq N}[l(n)]^{-q /(2-q)}\right)^{1-\frac{q}{2}}\|u\|^{q}+\frac{K(q)}{N^{\kappa}}\|u\|^{q} .
\end{aligned}
$$

This shows that (2.4) holds. 
Finally, we prove that $E$ is compactly embedded in $l^{q}\left(\mathbb{Z}, \mathbb{R}^{\mathcal{N}}\right)$. Let $\left\{u_{k}\right\} \subset E$ be a bounded sequence. Then by (2.1), there exists a constant $\Lambda>0$ such that

$$
\left\|u_{k}\right\|_{\infty} \leq \frac{1}{\sqrt[4]{(\beta+4 \alpha) \beta}}\left\|u_{k}\right\| \leq \Lambda, \quad k \in \mathbb{N} .
$$

Since $E$ is reflexive, $\left\{u_{k}\right\}$ possesses a weakly convergent subsequence in $E$. Passing to a subsequence if necessary, it can be assumed that $u_{k} \rightarrow u_{0}$ in $E$. It is easy to verify that

$$
\lim _{k \rightarrow \infty} u_{k}(n)=u_{0}(n), \quad \forall n \in \mathbb{Z}
$$

For any given number $\varepsilon>0$, we can choose $N_{\varepsilon}>0$ such that

$$
\frac{2^{q-1} K(q)}{N_{\varepsilon}^{\kappa}}\left\{[\sqrt[4]{(\beta+4 \alpha) \beta} \Lambda]^{q}+\left\|u_{0}\right\|^{q}\right\}<\varepsilon
$$

It follows from (2.8) that there exists $k_{0} \in \mathbb{N}$ such that

$$
\sum_{|n| \leq N_{\varepsilon}}\left|u_{k}(n)-u_{0}(n)\right|^{q}<\varepsilon \quad \text { for } k \geq k_{0}
$$

On the other hand, it follows from (2.3), (2.7) and (2.9) that

$$
\begin{aligned}
\sum_{|n|>N_{\varepsilon}}\left|u_{k}(n)-u_{0}(n)\right|^{q} & \leq 2^{q-1} \sum_{|n|>N_{\varepsilon}}\left(\left|u_{k}(n)\right|^{q}+\left|u_{0}(n)\right|^{q}\right) \\
& \leq \frac{2^{q-1} K(q)}{N_{\varepsilon}^{\kappa}}\left(\left\|u_{k}\right\|^{q}+\left\|u_{0}\right\|^{q}\right) \\
& \leq \frac{2^{q-1} K(q)}{N_{\varepsilon}^{\kappa}}\left\{[\sqrt[4]{(\beta+4 \alpha) \beta} \Lambda]^{q}+\left\|u_{0}\right\|^{q}\right\} \leq \varepsilon, \quad k \in \mathbb{N} .
\end{aligned}
$$

Since $\varepsilon$ is arbitrary, combining (2.10) with (2.11), we get

$$
\left\|u_{k}-u_{0}\right\|_{q}^{q}=\sum_{n \in \mathbb{Z}}\left|u_{k}(n)-u_{0}(n)\right|^{q} \rightarrow 0 \quad \text { as } k \rightarrow+\infty
$$

This shows that $\left\{u_{k}\right\}$ possesses a convergent subsequence in $l^{q}\left(\mathbb{Z}, \mathbb{R}^{\mathcal{N}}\right)$. Therefore, $E$ is compactly embedded in $l^{q}\left(\mathbb{Z}, \mathbb{R}^{\mathcal{N}}\right)$ for $1 \leq q \in(2 /(3-v), 2)$.

Lemma 2.3 Suppose that $L$ and $W$ satisfy $\left(\mathrm{L}_{v}\right)$ and $\left(\mathrm{W} 1^{\prime}\right)$. Then, for $u \in E$,

$$
\sum_{n \in \mathbb{Z}}|W(n, u(n))| \leq \phi_{1}(N)\|u\|^{\gamma_{1}}+\phi_{2}(N)\|u\|^{\gamma_{2}}, \quad N \geq N_{0},
$$

where

$$
\begin{gathered}
\kappa_{1}=\frac{(3-v) \gamma_{1}-2}{2}, \\
\kappa_{2}=\frac{(3-v) \gamma_{2}-2}{2} ;
\end{gathered}
$$




$$
\begin{aligned}
& \phi_{1}(N)=a_{1}\left[\left(\sum_{|n| \leq N}[l(n)]^{-\gamma_{1} /\left(2-\gamma_{1}\right)}\right)^{1-\frac{\gamma_{1}}{2}}+\frac{K\left(\gamma_{1}\right)}{N^{\kappa_{1}}}\right], \\
& \phi_{2}(N)=a_{2}\left[\left(\sum_{|n| \leq N}[l(n)]^{-\gamma_{2} /\left(2-\gamma_{2}\right)}\right)^{1-\frac{\gamma_{2}}{2}}+\frac{K\left(\gamma_{2}\right)}{N^{\kappa_{2}}}\right] .
\end{aligned}
$$

Proof For $N \geq N_{0}$, it follows from (2.4) and (W1') that

$$
\begin{aligned}
\sum_{n \in \mathbb{Z}}|W(n, u(n))| \leq & a_{1} \sum_{n \in \mathbb{Z}}|u(n)|^{\gamma_{1}}+a_{2} \sum_{n \in \mathbb{Z}}|u(n)|^{\gamma_{2}} \\
\leq & a_{1}\left[\left(\sum_{|n| \leq N}[l(n)]^{-\gamma_{1} /\left(2-\gamma_{1}\right)}\right)^{1-\frac{\gamma_{1}}{2}}+\frac{K\left(\gamma_{1}\right)}{N^{\kappa_{1}}}\right]\|u\|^{\gamma_{1}} \\
& +a_{2}\left[\left(\sum_{|n| \leq N}[l(n)]^{-\gamma_{2} /\left(2-\gamma_{2}\right)}\right)^{1-\frac{\gamma_{2}}{2}}+\frac{K\left(\gamma_{2}\right)}{N^{\kappa_{2}}}\right]\|u\|^{\gamma_{2}} \\
= & \phi_{1}(N)\|u\|^{\gamma_{1}}+\phi_{2}(N)\|u\|^{\gamma_{2}} .
\end{aligned}
$$

This shows (2.12) holds.

Lemma 2.4 Assume that $L$ and $W$ satisfy $\left(\mathrm{L}_{v}\right),\left(\mathrm{W} 1^{\prime}\right)$ and $\left(\mathrm{W} 2^{\prime}\right)$. Then the functional $f$ : $E \rightarrow \mathbb{R}$ defined by

$$
f(u)=\frac{1}{2}\|u\|^{2}-\sum_{n \in \mathbb{Z}} W(n, u(n)), \quad \forall u \in E
$$

is well defined and of class $C^{1}(E, \mathbb{R})$ and

$$
\begin{aligned}
\left\langle f^{\prime}(u), v\right\rangle= & \sum_{n \in \mathbb{Z}}[(p(n+1) \Delta u(n), \Delta v(n))+(L(n) u(n), v(n)) \\
& -(\nabla W(n, u(n)), v(n))], \quad \forall u, v \in E .
\end{aligned}
$$

Furthermore, the critical points off in $E$ are solutions of $(1.1)$ with $u( \pm \infty)=0$.

Proof Lemma 2.3 implies that $f$ defined by (2.16) is well defined on $E$. Next, we prove that (2.17) holds. By (W2'), one can choose an $M_{1}>0$ such that

$$
\varphi(|x|) \leq M_{1}|x|^{\gamma_{3}-1}, \quad \forall x \in \mathbb{R}^{\mathcal{N}},|x| \leq 1 .
$$

For any $u, v \in E$, there exists an integer $N_{1}>N_{0}$ such that $|u(n)|+|v(n)|<1$ for $|n|>N_{1}$. Then, for any sequence $\left\{\theta_{n}\right\}_{n \in \mathbb{Z}} \subset \mathbb{R}$ with $\left|\theta_{n}\right|<1$ for $n \in \mathbb{Z}$ and any number $h \in(0,1)$, by (W2'), (2.3) and Lemma 2.2, we have

$$
\begin{aligned}
& \sum_{n \in \mathbb{Z}} \max _{h \in[0,1]}\left|\left(\nabla W\left(n, u(n)+\theta_{n} h v(n)\right), v(n)\right)\right| \\
& \leq \sum_{|n| \leq N_{1}} \max _{h \in[0,1]}\left|\nabla W\left(n, u(n)+\theta_{n} h v(n)\right)\right||v(n)|
\end{aligned}
$$




$$
\begin{aligned}
& +\sum_{|n|>N_{1}} \max _{h \in[0,1]}\left|\nabla W\left(n, u(n)+\theta_{n} h v(n)\right)\right||v(n)| \\
\leq & \sum_{|n| \leq N_{1}} \max _{|x| \leq\|u\|_{\infty}+\|v\|_{\infty}}|\nabla W(n, x)||v(n)|+M_{1} \sum_{|n|>N_{1}}(|u(n)|+|v(n)|)^{\gamma_{3}-1}|v(n)| \\
\leq & \sum_{|n| \leq N_{1}} \max _{|x| \leq\|u\|_{\infty}+\|v\|_{\infty}}|\nabla W(n, x)||v(n)|+M_{1} \sum_{|n|>N_{1}}|v(n)|^{\gamma_{3}} \\
& +M_{1}\left(\sum_{|n|>N_{1}}|u(n)|^{\gamma_{3}}\right)^{1-\frac{1}{\gamma_{3}}}\left(\sum_{|n|>N_{1}}|v(n)|^{\gamma_{3}}\right)^{\frac{1}{\gamma_{3}}} \\
\leq & \sum_{|n| \leq N_{1}} \max _{|x| \leq\|u\|_{\infty}+\|v\|_{\infty}}|\nabla W(n, x)||v(n)|+\frac{M_{1} K\left(\gamma_{3}\right)}{N_{1}^{\kappa_{3}}}\left(\|u\|^{\gamma_{3}-1}+\|v\|^{\gamma_{3}-1}\right)\|v\| \\
< & +\infty,
\end{aligned}
$$

where $\kappa_{3}=\left[\gamma_{3}(3-v)-2\right] / 2>0$. Then by (2.16), (2.19) and Lebesgue's dominated convergence theorem, we have

$$
\begin{aligned}
\left\langle f^{\prime}(u), v\right\rangle & =\lim _{h \rightarrow 0^{+}} \frac{f(u+h v)-f(u)}{h} \\
& =\lim _{h \rightarrow 0^{+}}\left[\langle u, v\rangle+\frac{h\|v\|^{2}}{2}-\sum_{n \in \mathbb{Z}}\left(\nabla W\left(n, u(n)+\theta_{n} h v(n)\right), v(n)\right)\right] \\
& =\langle u, v\rangle-\sum_{n \in \mathbb{Z}}(\nabla W(n, u(n)), v(n)) \\
& =\sum_{n \in \mathbb{Z}}[(p(n+1) \triangle u(n), \Delta v(n))+(L(n) u(n), v(n))-(\nabla W(n, u(n)), v(n))] .
\end{aligned}
$$

This shows that (2.17) holds. Observe that for $u, v \in E$,

$$
\begin{aligned}
\sum_{n \in \mathbb{Z}} & {[(p(n+1) \triangle u(n), \Delta v(n))+(L(n) u(n), v(n))-(\nabla W(n, u(n)), v(n))] } \\
= & \sum_{n \in \mathbb{Z}}[\triangle(p(n) \triangle u(n-1), v(n))-(\triangle(p(n) \triangle u(n-1)), v(n))+(L(n) u(n), v(n)) \\
& -(\nabla W(n, u(n)), v(n))] \\
= & \sum_{n \in \mathbb{Z}}[(-\triangle(p(n) \triangle u(n-1))+L(n) u(n)-\nabla W(n, u(n)), v(n))]
\end{aligned}
$$

It follows from (2.17) and (2.20) that $\left\langle f^{\prime}(u), v\right\rangle=0$ for all $v \in E$ if and only if

$$
\triangle(p(n) \triangle u(n-1))-L(n) u(n)+\nabla W(n, u(n))=0, \quad \forall n \in \mathbb{Z} .
$$

So, the critical points of $f$ in $E$ are the solutions of system (1.1) with $u( \pm \infty)=0$.

Let us prove now that $f^{\prime}$ is continuous. Let $u_{k} \rightarrow u$ in $E$. Then there exists a constant $\delta>0$ such that

$$
\|u\| \leq \sqrt[4]{(\beta+4 \alpha) \beta} \delta, \quad\left\|u_{k}\right\| \leq \sqrt[4]{(\beta+4 \alpha) \beta} \delta, \quad k=1,2, \ldots
$$


It follows from (2.1) that

$$
\|u\|_{\infty} \leq \delta, \quad\left\|u_{k}\right\|_{\infty} \leq \delta, \quad k=1,2, \ldots
$$

By $\left(\mathrm{W} 2^{\prime}\right)$, one can choose an $M_{2}>0$ such that

$$
\varphi(|x|) \leq M_{2}|x|^{\gamma_{3}-1}, \quad \forall x \in \mathbb{R}^{\mathcal{N}},|x| \leq \delta
$$

From (2.3), (2.17), (2.21), (2.22), (2.23), (W2') and the Hölder inequality, we have

$$
\begin{aligned}
& \left|\left\langle f^{\prime}\left(u_{k}\right)-f^{\prime}(u), v\right\rangle\right| \\
& \leq\left|\sum_{n \in \mathbb{Z}}\left[\left(p(n+1)\left(\Delta u_{k}(n)-\triangle u(n)\right), \Delta v(n)\right)+\left(L(n)\left(u_{k}(n)-u(n)\right), v(n)\right)\right]\right| \\
& +\sum_{n \in \mathbb{Z}}\left|\left(\nabla W\left(n, u_{k}(n)\right)-\nabla W(n, u(n)), v(n)\right)\right| \\
& =\left|\left\langle u_{k}-u, v\right\rangle\right|+\sum_{n \in \mathbb{Z}}\left|\left(\nabla W\left(n, u_{k}(n)\right)-\nabla W(n, u(n)), v(n)\right)\right| \\
& \leq\left\|u_{k}-u\right\|\|v\|+\sum_{|n| \leq N}\left|\nabla W\left(n, u_{k}(n)\right)-\nabla W(n, u(n))\right||v(n)| \\
& +\sum_{|n|>N}\left(\left|\nabla W\left(n, u_{k}(n)\right)\right|+|\nabla W(n, u(n))|\right)|v(n)| \\
& \leq o(1)+M_{2} \sum_{|n|>N}\left(\left|u_{k}(n)\right|^{\gamma_{3}-1}+|u(n)|^{\gamma_{3}-1}\right)|v(n)| \\
& \leq o(1)+M_{2}\left(\sum_{|n|>N}\left|u_{k}(n)\right|^{\gamma_{3}}\right)^{1-\frac{1}{\gamma_{3}}}\left(\sum_{|n|>N}|v(n)|^{\gamma_{3}}\right)^{\frac{1}{\gamma_{3}}} \\
& +M_{2}\left(\sum_{|n|>N}|u(n)|^{\gamma_{3}}\right)^{1-\frac{1}{\gamma_{3}}}\left(\sum_{|n|>N}|v(n)|^{\gamma_{3}}\right)^{\frac{1}{\gamma_{3}}} \\
& \leq o(1)+\frac{M_{2} K\left(\gamma_{3}\right)}{N^{\kappa_{3}}}\left(\left\|u_{k}\right\|^{\gamma_{3}-1}+\|u\|^{\gamma_{3}-1}\right)\|v\| \\
& =o(1), \quad k \rightarrow+\infty, N \rightarrow+\infty, \forall v \in E,
\end{aligned}
$$

which implies the continuity of $f^{\prime}$. The proof is complete.

Lemma 2.5 [10] Let $E$ be a real Banach space and let $f \in C^{1}(E, \mathbb{R})$ satisfy the (PS)condition. Iff is bounded from below, then $c=\inf _{E} f$ is a critical value off .

Lemma 2.6 [11] Let $E$ be a real Banach space, let $f \in C^{1}(E, \mathbb{R})$ with $f$ even, bounded from below, and satisfying the (PS)-condition. Suppose that $f(0)=0$, there is a set $K \subset E$ such that $K$ is homeomorphic to $S^{k-1}$ by an odd map, and $\sup _{K} f<0$. Then $f$ possesses at least $k$ distinct pairs of critical points. 


\section{Proofs of theorems}

Proof of Theorem 1.1 In view of Lemma 2.4, $f \in C^{1}(E, \mathbb{R})$. In what follows, we first show that $f$ is bounded from below. Choose $N_{2}>N_{0}$, it follows from (2.12) that

$$
\sum_{n \in \mathbb{Z}} W(n, u(n)) \leq \phi_{1}\left(N_{2}\right)\|u\|^{\gamma_{1}}+\phi_{2}\left(N_{2}\right)\|u\|^{\gamma_{2}}, \quad \forall u \in E .
$$

By (2.16) and (3.1), we have

$$
f(u)=\frac{1}{2}\|u\|^{2}-\sum_{n \in \mathbb{Z}} W(n, u(n)) \geq \frac{1}{2}\|u\|^{2}-\phi_{1}\left(N_{2}\right)\|u\|^{\gamma_{1}}-\phi_{2}\left(N_{2}\right)\|u\|^{\gamma_{2}} .
$$

Since $1<\gamma_{1}<\gamma_{2}<2$, (3.2) implies that $f(u) \rightarrow+\infty$ as $\|u\| \rightarrow+\infty$. Consequently, $f$ is bounded from below.

Next, we prove that $f$ satisfies the (PS)-condition. Assume that $\left\{u_{k}\right\}_{k \in \mathbb{N}} \subset E$ is a sequence such that $\left\{f\left(u_{k}\right)\right\}_{k \in \mathbb{N}}$ is bounded and $f^{\prime}\left(u_{k}\right) \rightarrow 0$ as $k \rightarrow+\infty$. Then by (2.1) and (3.2), there exists a constant $A>0$ such that

$$
\left\|u_{k}\right\|_{\infty} \leq \frac{1}{\sqrt[4]{(\beta+4 \alpha) \beta}}\left\|u_{k}\right\| \leq A, \quad k \in \mathbb{N} .
$$

So, passing to a subsequence if necessary, it can be assumed that $u_{k} \rightarrow u_{0}$ in $E$. It is easy to verify that

$$
\lim _{k \rightarrow+\infty} u_{k}(n)=u_{0}(n), \quad \forall n \in \mathbb{Z}
$$

Hence, we have by (3.3) and (3.4)

$$
\left\|u_{0}\right\|_{\infty} \leq A
$$

By virtue of $\left(\mathrm{W} 2^{\prime}\right)$, one can choose a $M_{3}>0$ such that

$$
\varphi(|x|) \leq M_{3}|x|^{\gamma_{3}-1}, \quad \forall x \in \mathbb{R}^{\mathcal{N}},|x| \leq A .
$$

For any given number $\varepsilon>0$, we can choose an integer $N_{3}>N_{0}$ such that

$$
\frac{K\left(\gamma_{3}\right)}{N_{3}^{\kappa_{3}}}\left\{[\sqrt[4]{(\beta+4 \alpha) \beta} A]^{\gamma_{3}}+\left\|u_{0}\right\|^{\gamma_{3}}\right\}<\varepsilon
$$

It follows from (3.4) and the continuity of $\nabla W(n, x)$ on $x$ that there exists $k_{0} \in \mathbb{N}$ such that

$$
\sum_{n=-N_{3}}^{N_{3}}\left|\nabla W\left(n, u_{k}(n)\right)-\nabla W\left(n, u_{0}(n)\right)\right|\left|u_{k}(n)-u_{0}(n)\right|<\varepsilon \quad \text { for } k \geq k_{0} .
$$

On the other hand, it follows from (3.3), (3.5), (3.6), (3.7) and (W2') that

$$
\begin{aligned}
& \sum_{|n|>N_{3}}\left|\nabla W\left(n, u_{k}(n)\right)-\nabla W\left(n, u_{0}(n)\right)\right|\left|u_{k}(n)-u_{0}(n)\right| \\
& \quad \leq \sum_{|n|>N_{3}}\left[\varphi\left(\left|u_{k}(n)\right|\right)+\varphi\left(\left|u_{0}(n)\right|\right)\right]\left(\left|u_{k}(n)\right|+\left|u_{0}(n)\right|\right)
\end{aligned}
$$




$$
\begin{aligned}
& \leq M_{3} \sum_{|n|>N_{3}}\left(\left|u_{k}(n)\right|^{\gamma_{3}-1}+\left|u_{0}(n)\right|^{\gamma_{3}-1}\right)\left(\left|u_{k}(n)\right|+\left|u_{0}(n)\right|\right) \\
& \leq 2 M_{3} \sum_{|n|>N_{3}}\left(\left|u_{k}(n)\right|^{\gamma_{3}}+\left|u_{0}(n)\right|^{\gamma_{3}}\right) \\
& \leq \frac{2 M_{3} K\left(\gamma_{3}\right)}{N_{3}^{\kappa_{3}}}\left(\left\|u_{k}\right\|^{\gamma_{3}}+\left\|u_{0}\right\|^{\gamma_{3}}\right) \\
& \leq \frac{2 M_{3} K\left(\gamma_{3}\right)}{N_{3}^{\kappa_{3}}}\left\{[\sqrt[4]{(\beta+4 \alpha) \beta} A]^{\gamma_{3}}+\left\|u_{0}\right\|^{\gamma_{3}}\right\} \\
& \leq 2 M_{3} \varepsilon, \quad k \in \mathbb{N} .
\end{aligned}
$$

Since $\varepsilon$ is arbitrary, combining (3.8) with (3.9), we get

$$
\sum_{n \in \mathbb{Z}}\left(\nabla W\left(n, u_{k}(n)\right)-\nabla W\left(n, u_{0}(n)\right), u_{k}(n)-u_{0}(n)\right) \rightarrow 0 \quad \text { as } k \rightarrow+\infty
$$

It follows from (2.17) that

$$
\begin{aligned}
& \left\langle f^{\prime}\left(u_{k}\right)-f^{\prime}\left(u_{0}\right), u_{k}-u_{0}\right\rangle \\
& \quad=\left\|u_{k}-u_{0}\right\|^{2}-\sum_{n \in \mathbb{Z}}\left(\nabla W\left(n, u_{k}(n)\right)-\nabla W\left(n, u_{0}(n)\right), u_{k}(n)-u_{0}(n)\right) .
\end{aligned}
$$

Since $\left\langle f^{\prime}\left(u_{k}\right)-f^{\prime}\left(u_{0}\right), u_{k}-u_{0}\right\rangle \rightarrow 0$, it follows from (3.10) and (3.11) that $u_{k} \rightarrow u_{0}$ in $E$. Hence, $f$ satisfies the (PS)-condition.

By Lemma 2.5, $c=\inf _{E} f(u)$ is a critical value of $f$, that is, there exists a critical point $u^{*} \in E$ such that $f\left(u^{*}\right)=c$.

Finally, we show that $u^{*} \neq 0$. Let $u_{*}\left(n_{0}\right)=(1,0, \ldots, 0)^{\top} \in \mathbb{R}^{\mathcal{N}}$ and $u_{*}(n)=0$ for $n \neq n_{0}$. Then by $\left(\mathrm{W} 1^{\prime}\right),\left(\mathrm{W} 3^{\prime}\right)$ and $(2.16)$, we have

$$
\begin{aligned}
f\left(s u_{*}\right) & =\frac{s^{2}}{2}\left\|u_{*}\right\|^{2}-\sum_{n \in \mathbb{Z}} W\left(n, s u_{*}(n)\right) \\
& =\frac{s^{2}}{2}\left\|u_{*}\right\|^{2}-W\left(n_{0}, s u_{*}\left(n_{0}\right)\right) \\
& \leq \frac{s^{2}}{2}\left\|u_{*}\right\|^{2}-\eta s^{\gamma_{4}}\left|u_{*}\left(n_{0}\right)\right|^{\gamma_{4}}, \quad 0<s<\delta_{0} .
\end{aligned}
$$

Since $1<\gamma_{4}<2$, it follows from (3.12) that $f\left(s u_{*}\right)<0$ for $s>0$ small enough. Hence $f\left(u^{*}\right)=c<0$, therefore $u^{*}$ is a nontrivial critical point of $f$, and so $u^{*}=u^{*}(n)$ is a nontrivial homoclinic solution of system (1.1). The proof is complete.

Proof of Theorem 1.2 In view of Lemma 2.4 and the proof of Theorem 1.1, $f \in C^{1}(E, \mathbb{R})$ is bounded from below and satisfies the (PS)-condition. It is obvious that $f$ is even and $f(0)=0$. In order to apply Lemma 2.6, we prove now that there is a set $K \subset E$ such that $K$ is homeomorphic to $S^{m-1}$ by an odd map, and $\sup _{K} f<0$. Let

$$
J=\left\{n_{1}, n_{2}, \ldots, n_{m}\right\}
$$


where $n_{1}<n_{2}<\cdots<n_{m}$. Define

$$
u_{i}(n)=\left\{\begin{array}{ll}
(1,0, \ldots, 0)^{\top} \in \mathbb{R}^{\mathcal{N}}, & n=n_{i}, \\
0, & n \neq n_{i} ;
\end{array} \quad i=1,2, \ldots, m\right.
$$

and

$$
E_{m}=\operatorname{span}\left\{u_{1}, u_{2}, \ldots, u_{m}\right\}, \quad K_{m}=\left\{u \in E_{m}:\|u\|=1\right\} .
$$

For any $u \in E_{m}$, there exist $\lambda_{i} \in \mathbb{R}, i=1,2, \ldots, m$ such that

$$
u(n)=\sum_{i=1}^{m} \lambda_{i} u_{i}(n) \quad \text { for } n \in \mathbb{Z}
$$

Then

$$
\|u\|_{\gamma_{4}}=\left(\sum_{n \in \mathbb{Z}}|u(n)|^{\gamma_{4}}\right)^{1 / \gamma_{4}}=\left(\sum_{i=1}^{m}\left|\lambda_{i}\right|^{\gamma_{4}}\left|u_{i}\left(n_{i}\right)\right|^{\gamma_{4}}\right)^{1 / \gamma_{4}}
$$

and

$$
\begin{aligned}
\|u\|^{2} & =\sum_{n \in \mathbb{Z}}[(p(n+1) \triangle u(n), \Delta u(n))+(L(n) u(n), u(n))] \\
& =\sum_{n \in \mathbb{Z}}\left(p(n+1) \sum_{i=1}^{m} \lambda_{i} \Delta u_{i}(n), \sum_{j=1}^{m} \lambda_{j} \Delta u_{j}(n)\right)+\sum_{i=1}^{m} \lambda_{i}^{2}\left(L\left(n_{i}\right) u_{i}\left(n_{i}\right), u_{i}\left(n_{i}\right)\right) \\
& =\sum_{i=1}^{m} \sum_{j=1}^{m} \lambda_{i} \lambda_{j} \sum_{n \in \mathbb{Z}}\left(p(n+1) \Delta u_{i}(n), \Delta u_{j}(n)\right)+\sum_{i=1}^{m} \lambda_{i}^{2}\left(L\left(n_{i}\right) u_{i}\left(n_{i}\right), u_{i}\left(n_{i}\right)\right) \\
& =F\left(\lambda_{1}, \lambda_{2}, \ldots, \lambda_{m}\right),
\end{aligned}
$$

where $F\left(\lambda_{1}, \lambda_{2}, \ldots, \lambda_{m}\right)$ is a quadratic form. Since

$$
F\left(\lambda_{1}, \lambda_{2}, \ldots, \lambda_{m}\right)=\left\|\sum_{i=1}^{m} \lambda_{i} u_{i}\right\|^{2} \geq 0, \quad \forall\left(\lambda_{1}, \lambda_{2}, \ldots, \lambda_{m}\right)^{\top} \in \mathbb{R}^{m}
$$

and

$$
F\left(\lambda_{1}, \lambda_{2}, \ldots, \lambda_{m}\right)=0 \quad \Leftrightarrow \quad \sum_{i=1}^{m} \lambda_{i} u_{i}(n) \equiv 0, \quad \forall n \in \mathbb{Z} \quad \Leftrightarrow \quad \lambda_{1}=\lambda_{2}=\cdots=\lambda_{m}=0 .
$$

Therefore, $F\left(\lambda_{1}, \lambda_{2}, \ldots, \lambda_{m}\right)$ is a positive definite quadratic form. It follows that there exists an invertible matrix $\mathcal{A} \in \mathbb{R}^{m \times m}$ such that

$$
\left(\mu_{1}, \mu_{2}, \ldots, \mu_{m}\right)^{\top}=\mathcal{A}\left(\lambda_{1}, \lambda_{2}, \ldots, \lambda_{m}\right)^{\top}, \quad F\left(\lambda_{1}, \lambda_{2}, \ldots, \lambda_{m}\right)=\sum_{i=1}^{m} \mu_{i}^{2}
$$


Since all the norms of a finite dimensional normed space are equivalent, there is a constant $c^{\prime}>0$ such that

$$
c^{\prime}\|u\| \leq\|u\|_{\gamma_{4}} \quad \text { for } u \in E_{m} .
$$

By (W1'), (W4'), (3.14), (3.16) and (3.19), we have

$$
\begin{aligned}
f(s u) & =\frac{s^{2}}{2}\|u\|^{2}-\sum_{n \in \mathbb{Z}} W(n, s u(n)) \\
& =\frac{s^{2}}{2}\|u\|^{2}-\sum_{i=1}^{m} W\left(n_{i}, s \lambda_{i} u_{i}\left(n_{i}\right)\right) \\
& \leq \frac{s^{2}}{2}\|u\|^{2}-\eta s^{\gamma_{4}} \sum_{i=1}^{m}\left|\lambda_{i}\right|^{\gamma_{4}}\left|u_{i}\left(n_{i}\right)\right|^{\gamma_{4}} \\
& =\frac{s^{2}}{2}\|u\|^{2}-\eta s^{\gamma_{4}}\|u\|_{\gamma_{4}}^{\gamma_{4}} \\
& \leq \frac{s^{2}}{2}\|u\|^{2}-\eta\left(c^{\prime} s\right)^{\gamma_{4}}\|u\|^{\gamma_{4}} \\
& =\frac{s^{2}}{2}-\eta\left(c^{\prime} s\right)^{\gamma_{4}}, \quad \forall u \in K_{m}, 0<s \leq \delta_{0}\left(\sum_{i=1}^{m}\left|\lambda_{i}\right|\right)^{-1} .
\end{aligned}
$$

For $1<\gamma_{4}<2,(3.20)$ implies that there exist $\varepsilon>0$ and $\sigma>0$ such that

$$
f(\sigma u)<-\varepsilon \quad \text { for } u \in K_{m} .
$$

Let

$$
K_{m}^{\sigma}=\left\{\sigma u: u \in K_{m}\right\}, \quad S^{m-1}=\left\{\left(\mu_{1}, \mu_{2}, \ldots, \mu_{m}\right)^{\top} \in \mathbb{R}^{m}: \sum_{i=1}^{m} \mu_{i}^{2}=1\right\} .
$$

Then it follows from (3.17) that

$$
K_{m}^{\sigma}=\left\{\sum_{i=1}^{m} \lambda_{i} u_{i}: F\left(\lambda_{1}, \lambda_{2}, \ldots, \lambda_{m}\right)=\sigma^{2}\right\} .
$$

By (3.18), we define a map $\psi: K_{m}^{\sigma} \rightarrow S^{m-1}$ as follows:

$$
\psi(u)=\sigma^{-1}\left(\mu_{1}, \mu_{2}, \ldots, \mu_{m}\right)^{\top}, \quad \forall u \in K_{m}^{\sigma} .
$$

It is easy to verify that $\psi: K_{m}^{\sigma} \rightarrow S^{m-1}$ is an odd homeomorphic map. On the other hand, by (3.21), we have

$$
f(u)<-\varepsilon \quad \text { for } u \in K_{m}^{\sigma} \text {, }
$$

and so $\sup _{K_{m}^{\sigma}} f \leq-\varepsilon<0$. By Lemma 2.4, $f$ has at least $m$ distinct pairs of critical points, and so system (1.1) possesses at least $m$ distinct pairs of nontrivial homoclinic solutions. The proof is complete. 


\section{Examples}

In this section, we give three examples to illustrate our results.

Example 4.1 In system (1.1), let $p(n)$ be an $\mathcal{N} \times \mathcal{N}$ real symmetric positive definite matrix for all $n \in \mathbb{Z}, L(n)=\left(1+\sin ^{2} n\right)|n|^{2 / 3} I_{\mathcal{N}}$, and let

$$
W(n, x)=\cos n|x|^{4 / 3}+\sin n|x|^{3 / 2} .
$$

Then $L(n)$ satisfies $\left(\mathrm{L}_{v}\right)$ with $v=4 / 3$, and

$$
\begin{aligned}
& \nabla W(n, x)=\frac{4 \cos n}{3}|x|^{-2 / 3} x+\frac{3 \sin n}{2}|x|^{-1 / 2} x, \\
& |W(n, x)| \leq|x|^{4 / 3}+|x|^{3 / 2}, \quad \forall(n, x) \in \mathbb{Z} \times \mathbb{R}^{\mathcal{N}}
\end{aligned}
$$

and

$$
|\nabla W(n, x)| \leq \frac{8|x|^{1 / 3}+9|x|^{1 / 2}}{6}, \quad \forall(n, x) \in \mathbb{Z} \times \mathbb{R}^{\mathcal{N}} .
$$

For any $m \in \mathbb{N}$, there exist $m$ integers $n_{i} \in \mathbb{Z}, i=1,2, \ldots, m$ such that

$$
\cos n_{i}>0, \quad \sin n_{i}>0, \quad i=1,2, \ldots, m .
$$

Let $J=\left\{n_{1}, n_{2}, \ldots, n_{m}\right\}$ and

$$
\eta=\min \left\{\cos n_{1}+\sin n_{1}, \cos n_{2}+\sin n_{2}, \ldots, \cos n_{m}+\sin n_{m}\right\}
$$

Then

$$
W(n, x) \geq \eta|x|^{3 / 2}, \quad \forall(n, x) \in J \times \mathbb{R}^{\mathcal{N}},|x| \leq 1 .
$$

These show that all conditions of Theorem 1.2 are satisfied, where

$$
1<\frac{4}{3}=\gamma_{1}=\gamma_{3}<\gamma_{2}=\gamma_{4}=\frac{3}{2}<2, \quad a_{1}=a_{2}=1, \quad \varphi(s)=\frac{8 s^{1 / 3}+9 s^{1 / 2}}{6} .
$$

By Theorem 1.2, system (1.1) has at least $m$ distinct pairs of nontrivial homoclinic solutions. Since $m$ is arbitrary, it follows that system (1.1) has infinitely many distinct pairs of nontrivial homoclinic solutions.

Example 4.2 In system (1.1), let $p(n)$ be an $\mathcal{N} \times \mathcal{N}$ real symmetric positive definite matrix for all $n \in \mathbb{Z}, L(n)=\left(1+\cos ^{2} n\right)|n| I_{\mathcal{N}}$, and let

$$
W(n, x)=\sin \left(n+|x|^{2}\right)\left(|x|^{3 / 2}-2|x|^{7 / 4}\right) .
$$

Then $L(n)$ satisfies $\left(\mathrm{L}_{v}\right)$ with $v=1$, and

$$
\begin{aligned}
\nabla W(n, x)= & \sin \left(n+|x|^{2}\right)\left(\frac{3}{2}|x|^{-1 / 2} x-\frac{7}{4}|x|^{-1 / 4} x\right) \\
& +2 \cos \left(n+|x|^{2}\right)\left(|x|^{3 / 2}-2|x|^{7 / 4}\right) x,
\end{aligned}
$$




$$
\begin{aligned}
& |W(n, x)| \leq|x|^{3 / 2}+2|x|^{7 / 4}, \quad \forall(n, x) \in \mathbb{Z} \times \mathbb{R}^{\mathcal{N}}, \\
& |\nabla W(n, x)| \leq \frac{6|x|^{1 / 2}+7|x|^{3 / 4}+8|x|^{5 / 2}+16|x|^{11 / 4}}{4}, \quad \forall(n, x) \in \mathbb{Z} \times \mathbb{R}^{\mathcal{N}} .
\end{aligned}
$$

For any $m \in \mathbb{N}$, there exist $m$ integers $n_{i} \in \mathbb{Z}, i=1,2, \ldots, m$ such that

$$
\sin n_{i}>0, \quad \sin \left(n_{i}+1\right)>0, \quad i=1,2, \ldots, m .
$$

Let $J=\left\{n_{1}, n_{2}, \ldots, n_{m}\right\}$ and

$$
\eta=\frac{1}{2} \min \left\{\sin n_{i}, \sin \left(n_{i}+1\right): i=1,2, \ldots, m\right\}
$$

Then

$$
W(n, x) \geq \eta|x|^{3 / 2}, \quad \forall(n, x) \in J \times \mathbb{R}^{\mathcal{N}},|x| \leq \frac{1}{256}
$$

These show that all conditions of Theorem 1.2 are satisfied, where

$$
\begin{aligned}
& 1<\frac{3}{2}=\gamma_{1}=\gamma_{3}<\gamma_{2}=\gamma_{4}=\frac{7}{4}<2 ; \quad a_{1}=1, \quad a_{2}=2 ; \quad \delta_{0}=\frac{1}{256} ; \\
& \varphi(s)=\frac{6 s^{1 / 2}+7 s^{3 / 4}+8 s^{5 / 2}+16 s^{11 / 4}}{4} .
\end{aligned}
$$

By Theorem 1.2, system (1.1) has at least $m$ distinct pairs of nontrivial homoclinic solutions. Since $m$ is arbitrary, it follows that system (1.1) has infinitely many distinct pairs of nontrivial homoclinic solutions.

Example 4.3 In system (1.1), let $p(n)$ be an $\mathcal{N} \times \mathcal{N}$ real symmetric positive definite matrix for all $n \in \mathbb{Z}, L(n)=\ln \left(3+n^{2}\right)|n|^{4 / 3} I_{\mathcal{N}}$, and let

$$
W(n, x)=\sin n \ln \left(1+|x|^{3 / 2}\right) .
$$

Then $L(n)$ satisfies $\left(\mathrm{L}_{v}\right)$ with $v=2 / 3$, and

$$
\begin{aligned}
& \nabla W(n, x)=\frac{3 \sin n}{2\left(1+|x|^{3 / 2}\right)}|x|^{-1 / 2} x, \\
& |W(n, x)| \leq|x|^{3 / 2}, \quad|\nabla W(n, x)| \leq \frac{3|x|^{1 / 2}}{2\left(1+|x|^{3 / 2}\right)}, \quad \forall(n, x) \in \mathbb{Z} \times \mathbb{R}^{\mathcal{N}} .
\end{aligned}
$$

For any $m \in \mathbb{N}$, there exist $m$ integers $n_{i} \in \mathbb{Z}, i=1,2, \ldots, m$ such that

$$
\sin n_{i}>0, \quad i=1,2, \ldots, m
$$

Let $J=\left\{n_{1}, n_{2}, \ldots, n_{m}\right\}$ and

$$
\eta=\min \left\{\frac{\sin n_{i}}{2}: i=1,2, \ldots, m\right\}
$$


Then

$$
W(n, x) \geq \eta|x|^{3 / 2}, \quad \forall(n, x) \in J \times \mathbb{R}^{\mathcal{N}},|x| \leq 1
$$

These show that all conditions of Theorem 1.2 are satisfied, where

$$
1<\gamma_{1}=\gamma_{2}=\gamma_{3}=\gamma_{4}=\frac{3}{2}<2, \quad a_{1}=1, \quad a_{2}=0, \quad \varphi(s)=\frac{3 s^{1 / 2}}{2\left(1+s^{3 / 2}\right)} .
$$

By Theorem 1.2, system (1.1) has at least $m$ distinct pairs of nontrivial homoclinic solutions. Since $m$ is arbitrary, it follows that system (1.1) has infinitely many distinct pairs of nontrivial homoclinic solutions.

\section{Competing interests}

The authors declare that they have no competing interests.

\section{Authors' contributions}

XL wrote the first draft and XT corrected and improved the final version. Both authors read and approved the final draft.

\section{Author details}

'Department of Mathematics, Huaihua College, Huaihua, Hunan 418008, P.R. China. ${ }^{2}$ School of Mathematical Sciences and Computing Technology, Central South University, Changsha, Hunan 410083, P.R. China.

\section{Acknowledgements}

The authors would like to express their thanks to the referees for their helpful suggestions. This work is partially supported by the NNSF (No. 11171351) of China and supported by Scientific Research Fund of Hunan Provincial Education Department (08A053) and supported by Hunan Provincial Natural Science Foundation of China (No. 11 JJ2005).

\section{Received: 3 October 2012 Accepted: 10 May 2013 Published: 30 May 2013}

\section{References}

1. Agarwal, RP: Difference Equations and Inequalities: Theory, Methods and Applications, 2nd edn. Dekker, New York (2000)

2. Ahlbrandt, CD, Peterson, AC: Discrete Hamiltonian Systems: Difference Equations, Continued Fraction and Riccati Equations. Kluwer Academic, Dordrecht (1996)

3. Wong, JSW: On the generalized Emden-Fowler equation. SIAM Rev. 17, 339-360 (1975)

4. Ma, M, Guo, ZM: Homoclinic orbits for second order self-adjoint difference equations. J. Math. Anal. Appl. 323(1), 513-521 (2006)

5. Ma, M, Guo, ZM: Homoclinic orbits and subharmonics for nonlinear second order difference equations. Nonlinear Anal. 67, 1737-1745 (2007)

6. Deng, XQ, Cheng, G: Homoclinic orbits for second order discrete Hamiltonian systems with potential changing sign. Acta Appl. Math. 103, 301-314 (2008). doi:10.1007/s10440-008-9237-z

7. Tang, $X H$, Lin, $X Y, X i a o, L$ : Homoclinic solutions for a class of second order discrete Hamiltonian systems. J. Differ. Equ. Appl. 16, 1257-1273 (2010)

8. Lin, XY, Tang, XH: Existence of infinitely many homoclinic orbits in discrete Hamiltonian systems. J. Math. Anal. Appl. 373, 59-72 (2011)

9. Tang, XH, Lin, XY: Existence and multiplicity of homoclinic solutions for second-order discrete Hamiltonian systems with subquadratic potential. J. Differ. Equ. Appl. 17, 1617-1634 (2011)

10. Mawhin, J, Willem, M: Critical Point Theory and Hamiltonian Systems. Applied Mathematical Sciences, vol. 74 Springer, New York (1989)

11. Rabinowitz, PH: Minimax Methods in Critical Point Theory with Applications to Differential Equations. CBMS Reg. Conf. Ser. in Math., vol. 65. Am. Math. Soc., Providence (1986) 